\title{
Incidencia de la violencia física en la población LGBT en Ecuador
}

\author{
Incidence of physical violence in the LGBT population in Ecuador
}

Recibido: 18 de julio de 2016 - Revisado: 7 de diciembre de 2016 - Aceptado: 05 de junio de 2017.

\author{
Héctor Alberto Botello-Peñaloza ${ }^{1}$ \\ Isaac Guerrero-Rincón ${ }^{2}$
}

\section{Resumen}

La violencia hacia algunos colectivos de género ha sido un fenómeno que en los últimos años se ha visibilizado en América Latina, no obstante, y a diferencia de los países desarrollados, la región no dispone de estudios sobre esta realidad e instrumentos para atenderla. En consecuencia, el objetivo del presente artículo es hallar los determinantes de la violencia física en Ecuador a través de una encuesta amplia de colectivos de lesbianas, gais, bisexuales y transexuales (LGBT). Los resultados muestran que un $50 \%$ de los encuestados han sido agredidos, siendo los transexuales con bajo nivel educativo los más afectados. Igualmente, el análisis cuantitativo encuentra diferencias entre regiones, etnias y grupo de referencia (homosexuales, bisexuales, transexuales, transgénero), sin embargo, también resalta los avances que ha hecho la sociedad ecuatoriana en este aspecto. Se concluye que las estrategias que sirvan para aumentar la inclusión de la población de todos los géneros dentro de las oportunidades que presente el Estado, contribuirán a la reducción de las tendencias violentas entre grupos.

\section{Palabras clave}

Exclusión, LGBT, identidad de género, discriminación, violencia.

\begin{abstract}
Inter-gender violence is a phenomenon that in recent years has become visible in Latin America, however, and unlike the developed countries, the region has no studies on this reality and instruments to improve it. Consequently, the objective of this paper is to find the determinants of physical violence in Ecuador through a broad survey of lesbian, gay, bisexual and transgender (LGBT) groups. The results show that $50 \%$ of survey respondents have been assaulted, focusing on transsexuals with low educational level being the most affected. Likewise, the quantitative analysis finds differences between regions, races and reference group (homosexuals, bisexuals, transsexuals, transgender), however, it also highlights the advances that Ecuadorian society has made in this regard. It is concluded that strategies that increase the inclusion of the population of all gender within the opportunities presented to the state will contribute to the reduction of violent tendencies between groups.
\end{abstract}

\section{Keywords}

LGBT, violence, gender identity, discrimination, exclusion.

\footnotetext{
${ }^{1}$ Economista de la Universidad Industrial de Santander, Bucaramanga, Colombia. Candidato a Magíster en Ciencias Económicas, Universidad Nacional de Colombia. Miembro del Grupo de Investigación sobre Desarrollo Regional y Ordenamiento Territorial.

Correo electrónico:

hbotellop@unal.edu.co.

(10) https://orcid.org/0000-0002-77952590

2 Magíster en Economía, Universidad Nacional de Colombia, Bogotá, Colombia. Profesor de la Universidad Industrial de Santander, Bucaramanga, Santander, Colombia. Miembro del Grupo de Investigación sobre Desarrollo Regional y Ordenamiento Territorial.

Correo electrónico:

iguerrin@uis.edu.co.

(60) https://orcid.org/0000-0001-57942742

Para citar este artículo use: BotelloPeñaloza, H., y Guerrero-Rincón, I. (2018). Incidencia de la violencia física en la población LGBT en Ecuador. Civilizar, 18(35), 129-138. doi: 10.22518/usergioa/jour/ccsh/2018.2/ a10
} 


\section{Introducción}

La violencia es una expresión que surge de la interacción entre los seres vivos, siendo esto un producto del poder ejercido de forma coaccionante o de los patrones culturales que se reproducen y se imponen socialmente, derivando en daños físicos, psicológicos, mentales o morales (Barrientos, Silva, Catalan, Gómez, y Longueira, 2010; Bravo, 2014). En las sociedades humanas, la violencia ha sido un fenómeno estudiado desde diferentes puntos de vista según los grupos a los cuales afecta (Anderson y Bushman, 2002; Pinker, 2007). Sin embargo, un nuevo enfoque trata de estudiar la violencia que va dirigida a los grupos orientados por su género, denominada violencia intragénero (Parra y Ramos, 2012).

El punto de partida de esta violencia son los imaginarios culturales expresados en roles sociales y sexuales preestablecidos que derivan en que no se acepten a personas que posean una orientación sexual y afectiva diferente a la que siempre se ha enseñado. En el ámbito urbano, este tipo de violencia se visibiliza de manera aguda dada la cercanía fuera de los espacios como el trabajo, el colegio, el barrio etc. en los que se desenvuelven las personas.
Para combatir las formas de violencia intragénero, la Organización de las Naciones Unidas (ONU) propuso la Declaración sobre los derechos humanos, orientación sexual e identidad de género. El primer informe divulgativo sobre este tema presenta un patrón común de violaciones de derechos hacia las personas LGBT, que son blanco de injusticias de extremistas religiosos, paramilitares, neonazis, ultranacionalistas, entre otros (Parra y Ramos, 2012). El informe del Movimiento de Liberación Homosexual (2007) señala que el prejuicio hacia las minorías sexuales no se ha alterado de forma significativa en los últimos tiempos, como tampoco las acciones discriminatorias que se derivan de dicho fenómeno. Sin embargo, algunos países han avanzado en su aceptación sobre otras tendencias de género.

Esto se puede confirmar al observar los resultados de la Encuesta Mundial de Valores ${ }^{1}$ (World Values Survey, WVS), a la pregunta sobre la justificación sobre la homosexualidad (siendo 1 nada justificado y 10 totalmente justificado). Para 2014 los habitantes de países latinoamericanos poseían en promedio la mejor opinión sobre la homosexualidad y entre 1981 y 2014 han aumentado en mayor medida su opinión (Figura 1) especialmente Chile y Argentina.

Figura 1.

Diferencias en las opiniones de los encuestados sobre la justificación sobre la homosexualidad

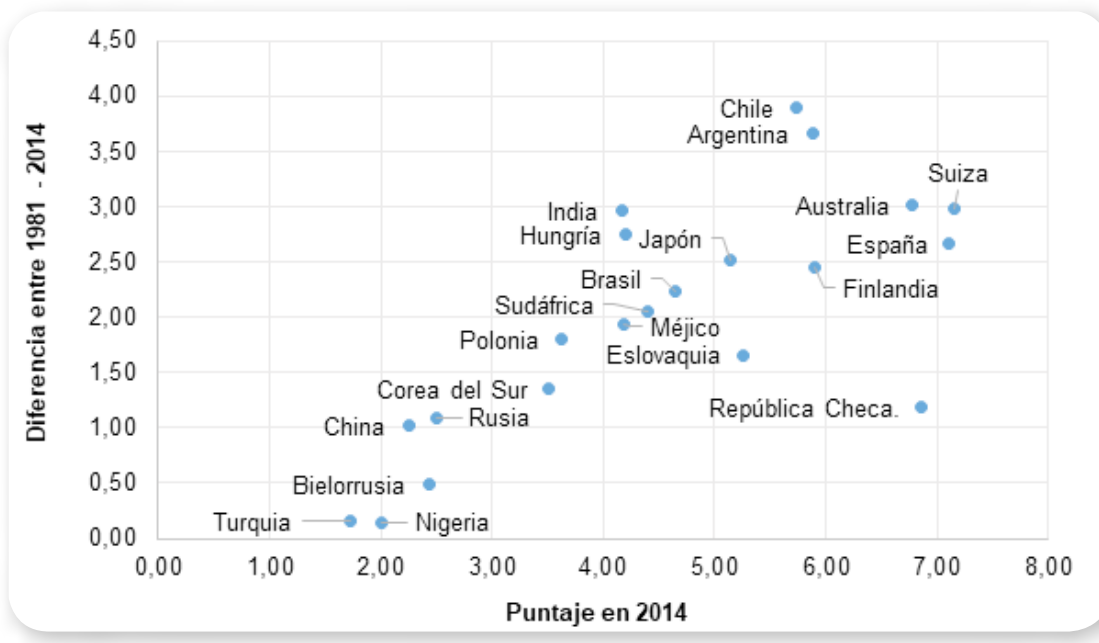

Fuente: World Values Survey, 1981 - 2014. 
En este sentido, en Latinoamérica son escasos los estudios cuantitativos llevados a cabo sobre las actitudes violentas hacia la población LGTB, sin embargo, la literatura evaluada muestra una tasa elevada de ataques de violencia física y discriminación. El estudio de la Corporación Chilena de Prevención del SIDA realizado a 148 personas con orientación LGTB, presenta resultados en los cuales el $38.1 \%$ afirmaron haber sufrido algún tipo de violencia por causa de su orientación sexual en los últimos cinco años y 77 (19.8\%) aseveraron haber sido detenidas alguna vez por la policía por su orientación sexual (CCHPS, 1997)².

En un análisis más reciente, la Comisión Interamericana de Derechos Humanos (CIDH) elaboró entre el 1 de enero de 2013 y el 31 de marzo de 2014, un cuestionario para obtener información sobre la violencia contra personas LGBTI, recopilando respuestas de 18 Estados Miembros de la $\mathrm{OEA}^{3}$. A través de este análisis se determinó que durante este período al menos 594 personas LGBT fueron asesinadas, cifras que incluyen 283 asesinatos de hombres gay y 282 asesinatos de personas transexuales. La CIDH también pudo identificar que las personas transexuales fueron más propensas a ser asesinadas con armas de fuego y sus cuerpos tienden a ser encontrados en las calles $u$ otros espacios públicos mientras que el resto se ubicó en lugares privados (hogares).

En este orden de ideas, la violencia ejercida hacia la comunidad LGBT es un problema social que debe ser evidenciado con el fin de generar un proceso de respeto, justicia, y buen trato, donde se les observe por sus cualidades y no por su condición sexual. Por lo anterior, el presente artículo parte de una investigación sobre la discriminación y la violencia hacia la población LGTB en el ecuador. Para lograr lo anterior el artículo encontró los determinantes de la violencia física en Ecuador a través de una encuesta amplia de colectivos de lesbianas, gais, bisexuales y transexuales (LGBT). Los resultados muestran que un 50\% de los encuesta- dos han sido agredidos, siendo los transexuales con bajo nivel educativo los más afectados. Se concluye que las estrategias que sirvan para aumentar la inclusión de la población de todos los géneros dentro de las oportunidades que presente el Estado, contribuirán a la reducción de las tendencias violentas entre grupos. A continuación, se describe detalladamente la metodología escogida para emprender este estudio.

\section{Metodología}

\section{Fuente de datos}

El siguiente trabajo analiza los determinantes que inciden en la violencia física que sufre la población de Gays, Lesbianas, Bisexuales, Transgénero, Transexuales, (LGBT) en el Ecuador por medio de un análisis descriptivo y econométrico de la base de datos sobre las Condiciones de Vida e Inclusión Social de Población LGBT en Ecuador del año 2012. Esta encuesta se realiza con el fin de elaborar un diagnóstico sobre las condiciones de vida, inclusión social, y cumplimiento de derechos humanos de este grupo poblacional. En la encuesta, el Instituto de Estadísticas del Ecuador (INEC) define a los conformantes de la población LGTB de la siguiente manera:

Gays: Término para identificar a hombres que asumen de manera abierta su atracción física, emocional y sexual por otros hombres.

Lesbianas: Término para reconocer a mujeres que aceptan de manera abierta su atracción física, emocional y sexual por otras mujeres.

Bisexuales: Mujeres o hombres que sienten atracción física, emocional y sexual por personas de ambos sexos.

Travestismo: Es una preferencia humana que se caracteriza por el uso de vestimenta, lenguaje, manierismos, etc., que en una determinada sociedad se consideran propios del género 
opuesto. Una persona puede travestirse de forma permanente, frecuente o esporádica.

La base de datos contiene información socioeconómica de los individuos a la vez que preguntas subjetivas sobre su situación de discriminación y segregación social. La reco lección de datos se realizó entre diciembre de 2012 y enero del 2013, en 10 ciudades del Ecuador, en las que previamente se realizó un mapeo de las organizaciones y colectivos LGBT. Las ciudades investigadas fueron: Guayaquil, Quito, Santa Elena, Salinas, Libertad, Manta, Portoviejo, Machala, Ibarra y Babahoyo. Se utilizó una técnica de muestreo no probabilístico a través del método Bola de Nieve ${ }^{4}$, que se caracteriza por la inexistencia de un marco muestral. Este método resulta eficaz para lograr el acercamiento y obtener la información de poblaciones ocultas.

El conteo final de la encuesta fue de 2.801 personas. La desagregación por grupos de edad evidencia que el $66,7 \%$ del colectivo se encuentra entre 20 y 34 años. Los datos enseñan que el $77,1 \%$ de las personas entrevistadas son solteras y el $16,1 \%$ declaran estar en unión libre.
Del total de las personas entrevistadas, aproximadamente el $41 \%$ alcanza al menos un año del nivel de instrucción superior universitario. Se obtiene que el $48,4 \%$ de la población entrevistada terminó el bachillerato y se encuentra cursando algún año en niveles superiores. Para el promedio de años de estudio, se considera solo a la población de 24 años y más. Así, se tiene un promedio 11,4 años de estudio, resultado relevante al considerar que es un dato superior al promedio de años de estudio a nivel nacional (9,6 años según el Censo de 2010)5.

\section{Figura 2.}

\section{Nivel educativo por orientación sexual}

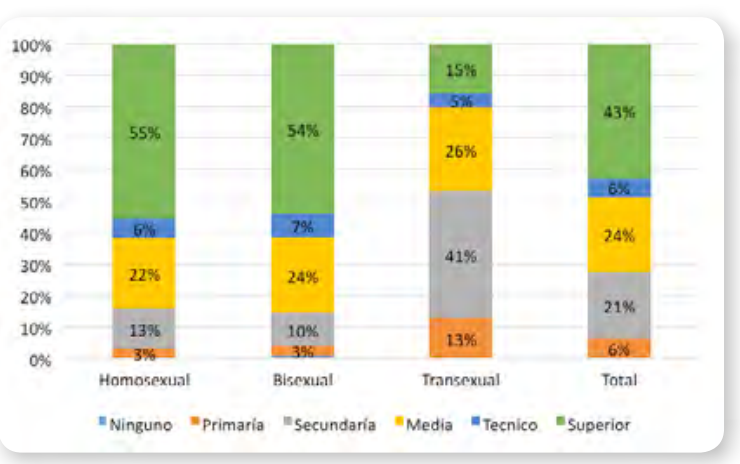

Fuente: Encuesta población LGTB. INEC Ecuador, 2012.

Tabla 1.

Número de personas entrevistadas por orientación y edad.

\begin{tabular}{|c|c|c|c|c|c|c|c|}
\hline Edad & \multicolumn{7}{|c|}{ Orientación sexual y/o identidad de género } \\
\hline & Gay & Lesbiana & Bisexual & Transfemenina & Transmasculino & Transexual & Total \\
\hline$<=18$ & 27 & 32 & 40 & 16 & 2 & 1 & 118 \\
\hline $19-23$ & 223 & 243 & 181 & 139 & 7 & 4 & 797 \\
\hline $24-28$ & 190 & 183 & 105 & 220 & 9 & 7 & 714 \\
\hline $29-33$ & 138 & 84 & 48 & 143 & 9 & 3 & 425 \\
\hline $34-38$ & 72 & 60 & 31 & 120 & 4 & 4 & 291 \\
\hline $39-43$ & 67 & 38 & 20 & 83 & 2 & 8 & 218 \\
\hline $44-48$ & 50 & 15 & 8 & 35 & 2 & 7 & 117 \\
\hline Más de 48 & 51 & 7 & 13 & 44 & 0 & 6 & 121 \\
\hline Total & $\mathbf{8 1 8}$ & $\mathbf{6 6 2}$ & $\mathbf{4 4 6}$ & $\mathbf{8 0 0}$ & $\mathbf{3 5}$ & $\mathbf{4 0}$ & $\mathbf{2 . 8 0 1}$ \\
\hline
\end{tabular}

Fuente: Encuesta población LGTB. INEC Ecuador, 2012. 


\section{Modelo}

El análisis de la violencia se realizará mediante un modelo econométrico que indagará sobre los determinantes que fijan una mayor probabilidad de que una persona de la población LGTB sea violentada físicamente. Frente a la falta de literatura guía en específico para la aplicación dentro de los grupos LGTB, el modelo implementado toma como base los escritos sobre violencia doméstica y de género en hogares y ciudades documentadas en Ribero y Sánchez (2004), Salas (2005), Díaz y Miranda (2009). En estos se implementa un modelo probabilístico para calcular los determinantes de la violencia hacia un grupo poblacional en particular.

En estos modelos, el fenómeno de estudio se configura como una variable que se indexa como 1 en el caso de que la persona haya sufrido de violencia física y 0 en el caso contrario, sucede que estas variables se denominan dicotómicas y para su análisis se ponen en práctica modelos de elección discreta.

Se supone que detrás de la variable dependiente en estos modelos, $Y$, se, se encuentra una variable no observable, $I$, que depende de un vector de variables independientes $X_{i}$ que toma ciertos valores si ha sobre pasado cierto lumbral, como se expresa a continuación:

$Y_{i}=\left\{\begin{array}{l}1 \text { si } I_{i}^{*}>0 \text { lo que ocurre cuando } X_{i} \beta+\varepsilon_{i}>0 \\ 0 \text { si } I_{i}^{*}<0 \text { lo que ocurre cuando } X_{i} \beta+\varepsilon_{i}<0\end{array}\right.$

La distribución del error $\varepsilon$, determina la clase de modelo a calcular, de la siguiente manera, si el error se comporta como una función de distribución uniforme, se utiliza el modelo lineal de probabilidad truncado; si es como una normal con media cero y varianza uno, el tratamiento será el de un probit; mientras que, si se supone que es mediante una curva logística, se trataría de un modelo logit. La hipótesis de que el umbral a superar por la variable latente sea cero, se puede modificar por cualquier otro valor sugiriéndose que el valor crítico sea el definido por el término constante (Pérez, 2005).

Bajo el primer enfoque el modelo probabilístico quedaría definido (Medina, 2003).

$$
\begin{aligned}
& \text { (1) } P_{i}=\operatorname{Prob}\left(Y_{i}=1\right)=\operatorname{Prob}\left(I_{i}^{*}\right) \\
& =\operatorname{Prob}\left(X_{i} \beta+\varepsilon_{i}>0\right)=f\left(X_{i} \beta\right)
\end{aligned}
$$

La eficiencia de estos modelos se estima en primera medida por la varianza de la variable dependiente captada o predicha por parte de las variables independientes, indicador denominado $\mathrm{R}^{2}$. En segundo lugar, se debe calcular cuál es el número de observaciones clasificadas correctamente en la característica analizada, un mayor porcentaje indica que el grado de robustez del modelo es mejor. Antes de realizar las interpretaciones de los resultados, hay que resolver diferentes situaciones sobre la naturaleza de los datos para una óptima calibración del modelo tales como la presencia de autocorrelación serial, heterocedasticidad, multicolinealidad (Pérez, 2005).

La ecuación de estimación, teniendo como base los criterios anteriores y el objetivo de esta investigación seria la función probabilística:

$$
Y=f(S, X) Y=\alpha+\beta_{i} X_{i}+\varepsilon
$$

Donde:

Y es la variable dicotómica dependiente;

1 es si la persona fue violentada;

0 en caso contrario;

$\beta$, mide el efecto marginal de cada una de las variables independientes;

$\mathrm{X}$, a la probabilidad de ser violentado,

$\varepsilon$ es el error de la estimación.

Según los trabajos documentados, se seleccionaron una serie de características con el objetivo de caracterizar el acto de violencia 
hacia la población LGTB, a continuación se enumeran las hipótesis planteadas:

H1: Ciertas poblaciones LGTB se ven más amenazadas con otras debido a la percepción dentro de la población.

H2: Las personas que se identifican abiertamente con su orientación pueden ser más violentadas que otras.

H3: Pueden observarse diferencias regionales y por etnias debido a la intensidad en los perjuicios sociales varía entre espacios y culturas.

Asimismo, se incluyeron otras variables de control para mejorar el proceso de cálculo del modelo que se estimaron en el modelo gracias al uso de variables dicotómicas.

\section{Resultados y análisis}

Este trabajo se enfoca a la violencia física ya que es la que la literatura científica ha logrado tratar cuantitativamente de mejor manera (Salas, 2005). Sin embargo, otras fuentes de sufrimiento que afectan a la población LGTB no son ignoradas, tal como se relaciona en la Tabla 2 , cerca del $75 \%$ de la población estudiada se ha visto sometida a situaciones de discriminación, $35 \%$ a exclusión y $50 \%$ a violencia física, siendo el grupo de transexuales los más afectados. Los resultados de la encuesta también apuntan a que los espacios públicos y privados ${ }^{6}$ son los sitios donde esta población se ve más afectada.

Tabla 2.

Porcentaje de población bajo los diferentes tipos de violencia

\begin{tabular}{l|c|c|c} 
Orientación & Discriminación & Exclusión & $\begin{array}{c}\text { Violencia } \\
\text { física }\end{array}$ \\
\hline Bisexual & $58.3 \%$ & $14.6 \%$ & $35.7 \%$ \\
\hline Gay & $65.0 \%$ & $23.0 \%$ & $44.3 \%$ \\
\hline Lesbiana & $73.7 \%$ & $26.4 \%$ & $47.7 \%$ \\
\hline Transexual & $93.7 \%$ & $65.7 \%$ & $66.5 \%$ \\
\hline Total general & $\mathbf{7 5 . 0 \%}$ & $\mathbf{3 5 . 8} \%$ & $\mathbf{5 0 . 7 \%}$
\end{tabular}

Fuente: Encuesta población LGTB. INEC Ecuador, 2012.
De acuerdo a esto, al evaluar las respuestas de la base de datos se seleccionaron 2.414 individuos para el análisis, posteriormente se realizaron las pruebas de chi cuadrado para probar el tipo de función con el cual se distribuía la variable dependiente, encontrándose que era mediante una representación de una curva logística. En este orden de ideas, la Tabla 3 muestra los resultados de las estimaciones del modelo logit con las elasticidades (efecto marginal) de cada una de las variables de control (independientes) sobre la probabilidad de la persona a ser violentada físicamente por su condición de LGTB.

En términos del ajuste global, el modelo resulta aceptable considerando que el valor del estadístico chi2 es muy significativo. A su vez, todas las variables introducidas presentaron niveles de significancia estadística del 10\% y según el R2 ajustado, el modelo consigue clasificar correctamente el $67 \%$ de los casos. Con esta comprobación de la robustez del modelo, se evidencian cada una de las hipótesis propuestas por el trabajo contrastándolas con el efecto que tengan las variables explicativas sobre la dependiente.

Para efectos de interpretación, el efecto marginal (elasticidad) en las variables categóricas, mide la probabilidad de manera relativa como comparación de una característica base, por ejemplo, la probabilidad de que una persona con educación universitaria sea violentada físicamente es un $36 \%$ menor a la de un individuo sin ninguna educación. Con estos parámetros también se pueden estimar las probabilidades absolutas de ser violentado si se posee o no una característica; esta última perspectiva es la utilizada en la mayor parte de las corroboraciones de las hipótesis planteadas.

En la hipótesis principal del trabajo se aprecia que los grupos de transexuales son el grupo con mayor incidencia de violencia física con un $14 \%$ por encima de la población Gay y un $26 \%$ por encima de los bisexuales. Este 
aspecto puede estar relacionado a las condiciones a las que se expone esta población al ser los más inclinados a realizar trabajos sexuales. De hecho, las personas que se encuentran ejerciendo esta actividad tienen un $20,5 \%$ más de probabilidad de ser violentadas físicamente que las que no.

En la segunda hipótesis, se aprecia que las personas que profesan su orientación mediante la pertenencia a grupos organizados de LGTB tienen hasta un $10 \%$ más de probabilidad de ser violentados. En el caso de las diferencias en la incidencia de la violencia por etnias, se aprecia una mayor probabilidad dentro de los indígenas ya que el resto de razas poseen efectos marginales negativos. En promedio, las personas de grupos LGTB analizadas tienen un 18\% más de posibilidad de ser agredidas al ser indígenas, que en el resto de la población. Sin embargo, el problema de confirmar lo anterior, es la baja representatividad de población LGTB encuestada para esta población.

Por regiones, la población de Machala posee la mayor probabilidad de ser afectada por hechos de violencia física que las del resto del país. En promedio, existe un 15\% más de

Tabla 3.

Resultados de las Estimaciones. Modelo Logit

\begin{tabular}{|c|c|c|c|c|c|}
\hline Variable & Característica Evaluada & Efecto Marginal & Error & Sig. (Prob.) & Característica base \\
\hline \multirow{2}{*}{ Grupo } & Bisexual & $-12.35 \%$ & $3.2 \%$ & 0.00 & \multirow{2}{*}{ Gay } \\
\hline & Transexual & $13.89 \%$ & $2.7 \%$ & 0.00 & \\
\hline Organización & No & $-10.28 \%$ & $2.6 \%$ & 0.00 & $\mathrm{Si}$ \\
\hline Educación & Universitario & $-36.04 \%$ & $16.7 \%$ & 0.05 & Ninguno \\
\hline \multirow{2}{*}{ Sexo biológico } & Mujer & $4.98 \%$ & $2.7 \%$ & 0.06 & \multirow{2}{*}{ Hombre } \\
\hline & Intersexual & $16.01 \%$ & $8.3 \%$ & 0.08 & \\
\hline \multirow{7}{*}{ Etnia } & Afrodescendiente & $-18.32 \%$ & $10.5 \%$ & 0.10 & \multirow{7}{*}{ Indígenas } \\
\hline & Negro & $-17.40 \%$ & $12.2 \%$ & 0.08 & \\
\hline & Mulato & $-15.61 \%$ & $9.8 \%$ & 0.03 & \\
\hline & Montubio & $-21.90 \%$ & $9.4 \%$ & 0.03 & \\
\hline & Mestizo & $-16.30 \%$ & $9.0 \%$ & 0.08 & \\
\hline & Blanco & $-23.31 \%$ & $8.9 \%$ & 0.02 & \\
\hline & Otro & $-33.23 \%$ & $10.6 \%$ & 0.02 & \\
\hline Trabajo Sexual & No & $-20.56 \%$ & $2.9 \%$ & 0.00 & $\mathrm{Si}$ \\
\hline \multirow{7}{*}{ Ciudad } & Babahoyo & $-17.53 \%$ & $5.6 \%$ & 0.00 & \multirow{7}{*}{ Machala } \\
\hline & Portoviejo & $-12.33 \%$ & $5.1 \%$ & 0.02 & \\
\hline & Manta & $-46.03 \%$ & $5.7 \%$ & 0.00 & \\
\hline & Quito & $-21.12 \%$ & $3.7 \%$ & 0.00 & \\
\hline & Santa Helena & $-23.87 \%$ & $5.7 \%$ & 0.00 & \\
\hline & Salinas & $-28.20 \%$ & $6.6 \%$ & 0.00 & \\
\hline & Guayaquil & $-7.71 \%$ & $3.8 \%$ & 0.05 & \\
\hline \multicolumn{2}{|c|}{ Observaciones } & 2.412 & \multicolumn{2}{|c|}{ Pseudo R2 } & 0.3873 \\
\hline \multicolumn{2}{|c|}{ Wald chi2(19) } & 229.36 & & $>\operatorname{chi} 2$ & 0.000 \\
\hline
\end{tabular}

Fuente: Cálculos del autor basado en la encuesta población LGTB. INEC Ecuador, 2012. 
probabilidad de ser violentado en esta ciudad por ser de orientación LGTB. En línea con lo anterior y según la encuesta de violencia de género, esta ciudad posee también la tasa de violencia de género e intrafamiliar más alta entre las principales ciudades del Ecuador (INEC, 2010).

Algo interesante a resaltar es que la edad del individuo no resultó significativa al determinar la violencia física, esto puede deberse al hecho de que la gran mayoría de los encuestados $(66,7 \%)$ se encuentra entre los 20 y 34 años y la discriminación de género puede ser un tema atemporal.

\section{Conclusiones}

La aceptación de la diversidad de género ha venido en aumento en todo el mundo a excepción de algunos países. Sin embargo, siguen existiendo focos de rechazo que han hecho que los gobiernos amplíen los derechos de estas poblaciones. En este sentido, este trabajo quiso evidenciar la incidencia de la violencia física dentro del colectivo de población LGTB en Ecuador a través de un análisis probabilístico de la encuesta realizada por el INEC en 2012. Los resultados apuntan a que la mitad de esta población ha sido atacada físicamente por su orientación, siendo el grupo de transexuales los de mayor incidencia de este fenómeno. Esta tasa es mayor a la que presentan otros países de la región, aunque la falta de estudios rigurosos no ha hecho posible una comparación más amplia.

Asimismo, la realización de trabajos sexuales, el tener un bajo nivel educativo o la pertenencia a una organización LGTB incrementan la probabilidad de incidencia de violencia física debido al factor de exposición que acarrean. También se encontró que existen diferencias regionales en la aceptación de estos colectivos, siendo la ciudad de Machala donde se encuentra una mayor incidencia de violencia física.
En respuesta a la presión de los grupos LGTB, Ecuador ha hecho más avances que ningún otro país en Latinoamérica en los últimos años a pesar de que en este país, hasta 1997, el ser homosexual era un delito. Entre las acciones se tiene, por ejemplo, el reconocimiento de la unión de hecho homosexual como un quinto estado dentro del Código Civil, además de la inclusión del género opcional en el año 2016 para el reconocimiento de la identidad de género de las personas transexuales. En términos judiciales, 475 notarías del país cuentan con información para la no discriminación por orientación sexual e identidad de género junto con cerca de 32.000 y 61.000 funcionarios públicos capacitados en derechos humanos y diversidad para el Ministerio de Justicia y del Interior respectivamente.

Para la salud de estos grupos, se han creado 104 centros inclusivos a nivel nacional, con 35.000 funcionarios capacitados en derechos humanos y diversidad. Asimismo, se va a realizar la inclusión de las variables de orientación sexual e identidad de género en el Registro Diario Automatizado (RDAACA) que funciona como un registro de salud adicional a la vida clínica de las personas. Además, el gobierno ha incrementado desde el 2012, los operativos de control a clínicas de adicciones con denuncias sobre incumplimiento de la norma sanitaria y casos de vulneración de derechos humanos por des-homosexualización.

Finalmente, si bien quedan muchos aspectos en donde la sociedad ecuatoriana debe avanzar para mejorar las condiciones de vida de la población LGTB, el reconocimiento de los derechos y la vigilancia efectiva en su protección son un paso adelante para lograr este objetivo. 


\section{Referencias}

Anderson, C. A., y Bushman, B. J. (2002). Human aggression. Annual Review of Psychology, 53, 27-51. doi:10.1146/ annurev.psych.53.100901.135231

Barrientos, J., Silva, J., Catalan, S., Gómez, F., y Longueira, J. (2010). Discrimination and victimization: parade for lesbian, gay, bisexual, and transgender (LGBT) pride, in Chile. Journal of homosexuality, 57(6), 760-775. doi: 10.1080/00918369.2010.485880

Bravo, F. L. (2014). El activismo LGBT ante la violencia intragénero. XXVI encuentros estatales LGTB, 14-16 noviembre. Valencia, España.

Corporación Chile de Prevención de Sida -CCHPS. (1997). De amores y sombras, poblaciones culturas homo y bisexuales en hombres de Santiago. Santiago. CCHPS.

Díaz, R., y Miranda-IEP, J. J. (2010). Aproximación del costo económico y determinantes de la violencia doméstica en el Perú. Economía y Sociedad, 75, 56-62.

Movimiento de Liberación Homosexual (2007). VI Informe Anual. Derechos humanos. Minorías sexuales chilenas. Hechos 2007. Recuperado de:

http://www.movilh.cl/documentos/VI-

INFORMEANUAL-ddhh-2007.pdf

Medina, E. (2003). Modelos de elección discreta. Publicaciones Económicas de la Universidad Autónoma de Madrid, España, 26.

Parra Piza, Y., y Ramos Melo, L. A. (2012). Huellas de la violencia simbólica en la población LGBT: Implicaciones sociales. Trabajo Social: Universidad Minuto de Dios. Bogotá, Colombia.

Pérez López, C. (2005). Métodos estadísticos avanzados con SPSS. Thompson. Madrid.
Pinker, S. (2007). A history of violence. New Republic, 236(4809), 18.

Ribero, R., y Sánchez, F. (2004). Determinantes, efectos y costos de la violencia intrafamiliar en Colombia. Documentos CEDE 002331. Universidad de Los Andes-Cede, Colombia.

Salas, L. (2005). Transmisión intergeneracional de la violencia intrafamiliar: evidencia para las familias colombianas. Desarrollo y Sociedad, (56), 285-337.

\section{Notas}

${ }^{1}$ Proyecto global de investigación social que explora los valores y opiniones de la gente y cómo estos cambian con el tiempo, además de su impacto social y político. Desde 1981 una red mundial de científicos sociales y politólogos llevan a cabo esta investigación, haciendo encuestas nacionales representativas en casi 100 países. La WVS es la única fuente de datos empíricos sobre actitudes y valores humanos que abarca a la mayoría de la población mundial (casi el 90\%).

${ }^{2}$ No se ha podido realizar una comparación más profunda dado que los datos oficiales sobre la violencia homofóbica son incompletos y las estadísticas oficiales escasas.

${ }^{3}$ Argentina, Bolivia, Brasil, Chile, Colombia, Ecuador, Estados Unidos, Guatemala, Honduras, Jamaica, México, Nicaragua, Panamá, Paraguay, Perú, Suriname, Uruguay y Venezuela.

${ }^{4}$ Más información del método de recolección y demás aspectos de la encuesta mirar el documento del INEC:

http://www.ecuadorencifras.gob.ec/ documentos/web-inec/Estadisticas_Sociales/ LGBTI/Metodologia_estudio_de_caso_ LGBTI-octubre2013.pdf 
5 Más información de descripción estadística de la encuesta la puede encontrar en: http://www.ecuadorencifras.gob.ec/ documentos/web-inec/Estadisticas Sociales/LGBTI/Analisis_situacion LGBTI.pdf
${ }^{6}$ Se consideran espacios públicos: calles, plazas, parques, malecones. Se consideran espacios privados: casas, restaurantes, bares, saunas, discotecas, centros comerciales, etc. 